\title{
Multispectral enhancement method to increase the visual differences of tissue structures in stained histopathology images
}

\author{
Pinky A. Bautista* and Yukako Yagi \\ Department of Pathology, Massachusetts General Hospital, Harvard Medical School, Boston, MA, USA
}

Received: June 26, 2012

Accepted: July 30, 2012

\begin{abstract}
In this paper we proposed a multispectral enhancement scheme in which the spectral colors of the stained tissuestructure of interest and its background can be independently modified by the user to further improve their visualization and color discrimination. The colors of the background objects are modified by transforming their $N$-band spectra through an $N x N$ transformation matrix, which is derived by mapping the representative samples of their original spectra to the spectra of their target colors using least mean square method. On the other hand, the color of the tissue structure of interest is modified by modulating the transformed spectra with the sum of the pixel's spectral residual-errors at specific bands weighted through an $N x N$ weighting matrix; the spectral error is derived by taking the difference between the pixel's original spectrum and its reconstructed spectrum using the first $M$ dominant principal component vectors in principal component analysis. Promising results were obtained on the visualization of the collagen fiber and the non-collagen tissue structures, e.g., nuclei, cytoplasm and red blood cells (RBC), in a hematoxylin and eosin (H\&E) stained image.
\end{abstract}

Keywords: Multispectral, enhancement, visualization, multispectral, detection, histopathology

\section{Introduction}

Color is the most accessible feature of an image for use in image interpretation [1]. In pathology diagnosis, color is a primary feature of stained tissue sections by which diagnostic interpretations are derived. There are many varieties of chemical stains that can be utilized to visualize the tissue structures' organization. For routine staining, however, hematoxylin and eosin (H\&E) staining is the most popular. This stain mainly differentiates the nuclei areas from the cytoplasm areas. When abnormal changes in the ratio between the cytoplasm and nuclei areas are observed other staining techniques, i.e., special stains or immunohistochemical stain, are employed to further probe the

*Corresponding author: Pinky A. Bautista, MGH Pathology Imaging and Communication Technology (PICT) Center, $101 \mathrm{Mer}-$ rimac St. Suite 820, Boston, MA 02114, USA. Tel.: +1 617643 7915; Fax: +1 617643 7901; E-mail: pbautista@partners.org. cause of the abnormality. Due to the popularity of the $\mathrm{H} \& \mathrm{E}$ staining, a number of investigations have been done to digitally simulate its staining effect and to investigate the discrimination of $H \& E$ stained tissue structures which are ordinarily differentiated with the use of other types of stain [2-8]. The success of these research endeavors would eventually allow pathologists to quantify the distribution of tissue structures in the absence of special stains. In effect, the cost of staining and the risk of losing the area of interest in tissue re-cuts are reduced.

Interest on the application of multispectral imaging, which is widely used in remote sensing applications, to pathology diagnosis has grown over the years. Results on several pilot studies on the utility of multispectral imaging to improve the visualization of tissue structures and accuracy of quantitative histopathology image analysis showed promise [2-15]. A multispectral image acquisition system captures the same scene at different spectral wavelength using 
narrowband spectral filters producing a stack of $N$ grey-level spectral images, where $N$ corresponds to the number of narrowband spectral filters employed by the imaging system. Compared to conventional RGB color image, a multispectral image carries more spectral color information. Hence, pertinent to histopathology image analysis where stained tissue structures are mainly discriminated through their staining colors, the use of multispectral imaging rather than the conventional RGB imaging, which utilizes wideband spectral color filters, is expected to be more effective.

Image enhancement is a valuable tool to improve the visual color discrimination between the different classes of objects in the image. It is typically employed to pre-process images to improve the accuracy of the quantitative image analyses [16-20]. Multispectral enhancements are implemented to either improve our visual perception of the objects in a multispectral image or to particularly detect an object which is not visually perceptible [2-8, 14-15]. When the objective of the enhancement is to emphasize the spectral-color difference between the object of interest and its background the visual clarity of the different objects in the background are not generally given attention. In stained histopathology images, the background objects in an image could consist of tissue structures whose features are also relevant in the diagnosis. Hence an enhancement scheme that improves the color discrimination between the structures in the background while at the same time enhances the tissue structure of interest is beneficial either for manual or automatic image analysis task.

In this paper we introduce a spectral enhancement scheme which, aside from bringing out improved color difference between the tissue structure of interest and its background, provides an option for users to change the colors of the background objects to either increase their color discrimination or to simulate their staining patterns as reactions to a particular chemical stain. The details of the proposed multispectral enhancement are discussed in Section 2.The results of our experiments on the visualization of collagen fiber, which is ordinarily emphasize using special stain, i.e., Masson's trichrome stain, from an H\&E stained tissue image are presented in Section 3. Evaluation of the enhancement results are also presented in this section. Discussions about the results, limitations of the present multispectral enhancement and thoughts about future works are presented in Section 4. Our conclusion is presented in Section 5.

\section{Method}

\subsection{Previous multispectral enhancement method}

We based our proposed multispectral enhancement methodology on the spectral enhancement introduced by Mitsui et al. [15]. If $\mathbf{t}_{o}$ is the original $N$-band spectral transmittance of the multispectral pixel, its enhanced spectral transmittance $\mathbf{t}_{e}$ is given in the following form:

$$
\begin{aligned}
& \mathbf{t}_{e}=\mathbf{t}_{o}+\mathbf{W} \boldsymbol{\varepsilon} \\
& \boldsymbol{\varepsilon}=\mathbf{t}_{o}-\hat{\mathbf{t}}_{o}
\end{aligned}
$$

In the above equations $\mathbf{t}_{o}, \mathbf{t}_{e}$, and $\boldsymbol{\varepsilon}$ are all $N \mathrm{x} 1$ column vectors while $\mathbf{W}$ is an $N X N$ matrix. The notation $\boldsymbol{\varepsilon}$ is referred as the spectral residual-error and is determined by taking the difference between the original spectrum and the reference spectrum, $\hat{\mathbf{t}}_{o}$, Eq. 2. The notation $\mathbf{W}$, on the other hand, is defined as the weighting matrix whose elements function as weighting factors to the spectral residual-errors at different bands. Hence, through the matrix $\mathbf{W}$ the amount of spectral error feed back to the original spectrum can be controlled. Furthermore, the reference spectrum $\hat{\mathbf{t}}_{o}$ in Eq. 2 is the pixel's original spectrum reconstructed using $M$ dominant principal component vectors in principal component analysis (PCA) and calculated as follows:

$$
\hat{\mathbf{t}}_{o}=\mathbf{V} \mathbf{V}^{T} \mathbf{t}_{o}+\left[\mathbf{I}-\mathbf{V} \mathbf{V}^{T}\right] \overline{\mathbf{t}}
$$

where $\overline{\mathbf{t}}$ is the average spectrum of the spectral samples used in the principal component analysis (PCA); $\mathbf{I}$ is an $N x N$ identity matrix; and $\mathbf{V}$ is an $N x N$ matrix whose $j$ th column correspond to the $j$ th principal component vector, $\mathbf{V}_{m}$ :

$$
[\mathbf{V}]_{j}=\left\{\begin{array}{l}
\mathbf{v}_{j} j=<M \\
0
\end{array}\right.
$$

The enhancement formulation in Eq. 1 modifies the color of the object of interest, but not the color of the background objects. That is, in the previous enhancement formulation the users do not have options to modify the colors of the background objects. In addition, the users cannot freely specify the enhancement color for the object of interest as it is directly tied to the band/s at which the spectral residual-error, $\boldsymbol{\varepsilon}$, of the object peaks. For example, if the object of interest's error peaks at $550 \mathrm{~nm}$ then its enhancement color is associated to the spectral color of this wavelength. These limitations are addressed in the proposed 
multispectral enhancement scheme discussed in the next section.

\subsection{Proposed multispectral enhancement method}

There are three phases in the proposed multispectral enhancement method: (i) modification of the pixels' spectral colors; (ii) detection of the object of interest; and (iii) multispectral enhancement. In the spectral color modification phase the user first manually selects the background objects whose colors he/she desires to modify in the enhancement. After which the user specifies the envisioned colors of these objects in the enhanced image. The $N$-band spectra of the objects' original and target colors, which could either be calculated or measured, are also provided by the user at this phase. In this work the relation between the $N$-band spectra of the original and target colors is considered linear such that we can derive an $N x N$ matrix describing such relation by least mean-square pseudoinverse method [5]. In the object detection phase, the spectral residual-error configurations of the different objects in the sample images are investigated. The bands at which the spectral error of the object of interest is large compared to background objects (or object of non-interest) are noted. The errors at these bands are amplified to emphasize the detected object of interest. The last phase of the present multispectral enhancement methodology is the superposition of the results in phases (i) and (ii) to produce the enhanced spectrum of the multispectral pixel. This process is repeated for all the multispectral pixels to produce an enhanced multispectral image wherein we can observe clearer color discrimination between the object of interest and its background. The processes involve in each phase are discussed at length in the following sub-sections.

\subsubsection{Modification of the background objects' spectral-color}

Two sets of spectral data are specified by the user at this stage: (i) the spectra of the objects' original colors; and (ii) the spectra of the objects' target colors, which are the colors envisioned by user. The NXN transformation matrix that characterizes the relation between the original and target spectra is derived and kept for use in the actual multispectral enhancement procedure.

Let $\left\{\mathbf{f}_{c}, \mathbf{g}_{c}\right\}$ be a pair of $N x 1$ column vectors which respectively represent the $N$-band spectra of the original and target colors of the $c$ th background object. Furthermore, let $\mathbf{Q}_{g}$ be the $N x N$ transformation matrix that maps $\mathbf{f}_{c}$ to $\mathbf{g}_{c}$ such that:

$$
\mathbf{g}_{c}=\mathbf{Q}_{g} \mathbf{f}_{c}
$$

The solution for $\mathbf{Q}_{g}$ can be found by applying the linear mean-square method to the spectral samples of the $C$ number of background objects, i.e., $c=1,2, \ldots C$.

$$
\mathbf{Q}_{g}=\mathbf{G F}^{+}
$$

where $\mathbf{F}$ and $\mathbf{G}$ are $N X C$ matrices which hold the representative spectra of the original and target colors of the $C$ objects, respectively; and $\mathbf{F}^{+}$is the pseudoinverse of matrix $\mathbf{F}$.

\subsubsection{Detection of object of interest}

The object of interest is detected based on its acquired spectral residual-error. With Eq. 3, we can express the spectral residual-error, $\boldsymbol{\varepsilon}$, in Eq. 2 into the following form:

$$
\boldsymbol{\varepsilon}=\left[\mathbf{I}-\mathbf{V} \mathbf{V}^{T}\right] \mathbf{t}_{o}-\left[\mathbf{I}-\mathbf{V} \mathbf{V}^{T}\right] \overline{\mathbf{t}}
$$

The result of Eq. 7 is an $N x l$ column vector which reflects the error characteristics of a multispectral object at different spectral bands. The spectral residualerror of an object varies across the different spectral bands. At some bands the error of the object could be small while at some bands it could be large. In previous works $[4,5]$ the spectral residual-error characteristics of the various objects in the image were investigated to gain knowledge on the spectral bands at which the spectral errors' magnitudes of the object of interest are larger compared to the background objects. These bands were considered as the bands for enhancement since at these bands the object of interest can be fairly detected. In order to give more emphasis to the detected objects and hence provide clearer visualization of their presence, the errors at these bands are amplified. In this procedure, the resulting color of the detected object is tied to the spectral colors of the bands for enhancement. That is, the user doesn't have the flexibility to specify the enhancement color of the detected object. This can be mitigated by re-assigning the elements of the spectral residual-error vector in Eq. 7 to a constant. Let $\boldsymbol{\gamma}$ be an $N \mathrm{x} 1$ vector representing the modified spectral residual-error whose ith element, $i=1,2, . . N$, is computed as follows: 


$$
\gamma_{i}=\sum_{j \in b_{o}} \varepsilon_{j} \forall i
$$

By incorporating Eq. 8 to the original spectral error expression in Eq. 7 we will have:

$$
\begin{aligned}
& \gamma=\mathbf{A}\left[\mathbf{I}-\mathbf{V} \mathbf{V}^{T}\right] \mathbf{t}_{o}-\mathbf{A}\left[\mathbf{I}-\mathbf{V} \mathbf{V}^{T}\right] \overline{\mathbf{t}} \\
& {[\mathbf{A}]_{i j}= \begin{cases}1 j \in \boldsymbol{b}_{o}, & \forall i \\
0 & \text { otherwise }\end{cases} }
\end{aligned}
$$

where $\mathbf{A}$ is an $N x N$ matrix, and the variable $\boldsymbol{b}_{o}$ is a set of band numbers which correspond to the spectral bands at which the spectral errors of the object of interest are large, i.e., bands for enhancement. Since the magnitude of the spectral residual-error, Eq. 9, no longer varies between spectral bands, the bands for enhancement can be defined by the user by assigning appropriate weighting factors to the spectral errors at the selected spectral bands. Let us define the $N x N$ weighting matrix $\mathbf{W}$ in Eq. 1 as follows:

$$
[\mathbf{W}]_{i j}= \begin{cases}k_{i} & i=j \in \boldsymbol{b}_{d} \\ 0 & \text { otherwise }\end{cases}
$$

where $k_{i} \in \Re$, and is specified by the user. Moreover, $\boldsymbol{b}_{d}$ is the set containing the user-defined bands for enhancement. Introducing $\mathbf{W}$ in Eq. 11 to the spectral residual-error in Eq. 9 results to the following form:

$$
\mathbf{o}_{d}=\mathbf{W} \boldsymbol{\gamma}
$$

where $\mathbf{o}_{d}$ is an $N x 1$ vector representing the weighted spectral residual-error of a multispectral pixel. The desired color for enhancement is obtained by boosting the spectral errors at the bands specified by the user in $\boldsymbol{b}_{d}$, and suppressing the errors at other bands. Hence, through the weighting matrix $\mathbf{W}$ the color for enhancement can be controlled by the user. In should be noted that while the spectral bands in $\boldsymbol{b}_{o}$ are identified from the spectral residual-error configuration of the object of interest, those in $\boldsymbol{b}_{d}$ are freely specified by the user.

\subsubsection{Multipectral enhancement}

The proposed multispectral enhancement scheme is derived by introducing modifications to Eq. 1. The $N X N$ matrix $\mathbf{Q}_{g}$ determined from Eq. 6 is introduced and the second term of the equation is replaced with the expression given in Eq. 12. The proposed enhancement scheme can then be expressed as follows:

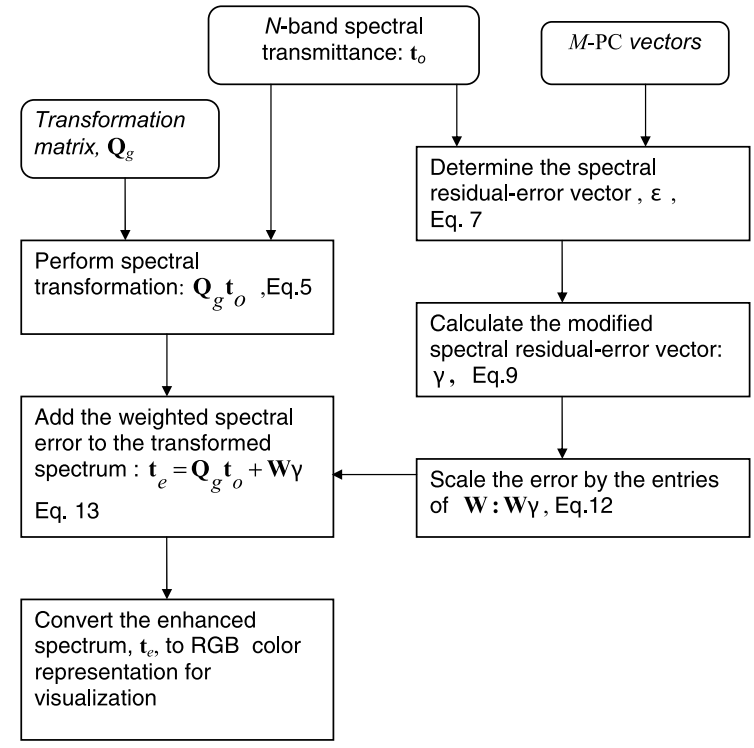

Fig. 1. Process flow of the proposed multispectral enhancement scheme.

$$
\begin{aligned}
\mathbf{t}_{e}= & {\left[\mathbf{W A}\left(\mathbf{I}-\mathbf{V} \mathbf{V}^{T}\right)+\mathbf{Q}_{g}\right] \mathbf{t}_{o} } \\
& -\mathbf{W A}\left[\mathbf{I}-\mathbf{V} \mathbf{V}^{T}\right] \overline{\mathbf{t}}
\end{aligned}
$$

The present modifications broaden the functionality of the enhancement scheme which was originally introduced in [15]. These modifications allow the user to not only control the enhancement color of the object of interest but also to re-define the colors of the background objects. Equation 13 generates the same results as Eq. 1 when and if the $N x N$ matrix $\mathbf{Q}_{g}$ is an identity matrix and $\boldsymbol{\gamma}=\boldsymbol{\varepsilon}$. In order for the $N$-band enhanced spectrum $\mathbf{t}_{e}$ to be observed on ordinary color display, the spectrum is transformed to its equivalent RGB color values [21].

The actual implementation of the proposed multispectral enhancement method requires that the $N x N$ matrices $\mathbf{Q}_{g}, \mathbf{V}, \mathbf{W}$, and $\mathbf{A}$ must all be determined offline. Spectral samples of the various objects in the image, i.e., object of interest and its background objects, are needed to calculate the entries of matrices $\mathbf{Q}_{g}$ and $\mathbf{V}$. Information on the enhancement band set, $\boldsymbol{b}_{o}$, is also needed to effectively calculate the modified spectral residual-error, Eq. 9, and determine the spatial location of the non-zero entries for the matrix $\mathbf{A}$, Eq. 11. The user-defined bands for enhancement $\boldsymbol{b}_{d}$ should also be known to define the weighting matrix W, Eq. 12. The process flow of enhancing an $N$-band spectral transmittance $\mathbf{t}_{o}$ is illustrated in Fig. 1. 


\section{Experimental results}

\subsection{Multispectral images used}

We used the microscopic multispectral imaging system developed by Olympus, Japan to capture 13 sets of stained liver tissue images. Each set is composed of H\&E and Masson's trichrome stained multispectral images of the tissue. The H\&E and Masson's trichrome stained sections are contiguous so that the pair of images in a set shares similar structural makeup. The spatial dimension of each multispectral image
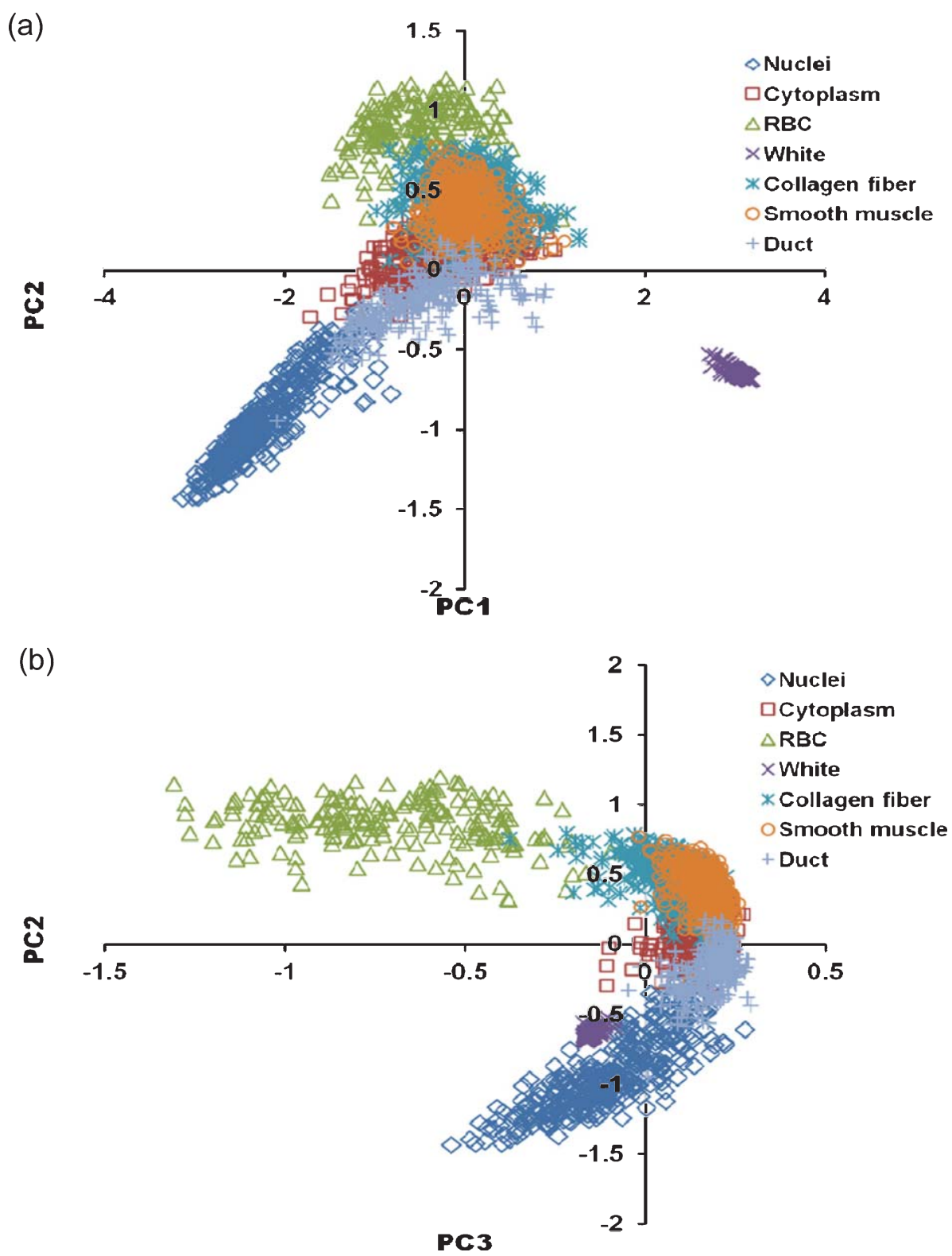

Fig. 2. Spectral distribution of the different tissue components, which were identified from the captured images, projected onto the principal component (PC) axes. The distribution show similarly stained tissue structures, such as the smooth muscle and collagen fiber, could be difficult to differentiate using their staining colors: (a) projection of the spectral samples onto the 1st and 2nd PC axes; (b) projection of the spectral samples onto the 2nd and 3rd PC axes. The number of spectral samples for each tissue component is as follows: Nucleus = 350; Cytoplasm =450; $\mathrm{RBC}=200$; Collagen fiber $=425$; Smooth muscle $=450$ and Duct $=300$. And the white areas are represented with 350 spectral samples. 


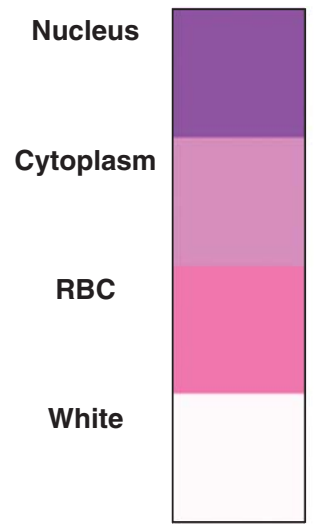

(a)

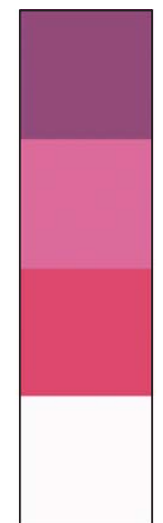

(b) (c)

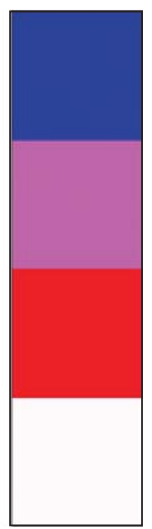

Fig. 3. Color patches illustrating the original and target colors of the background tissue components, i.e., nucleus, cytoplasm, and red blood cells (RBC), whose spectral transmittance were selected to derive the transformation matrix. (a) Original colors; and (b) and (c) illustrate the target colors. The colors in (b) mimics the staining colors of the tissue components when stained with Masson's trichrome, while those in (c) were freely specified by the user.

is $1434 \times 1050$ pixels and its spectral dimension is $N=63$. The 63 spectral band images have spectral resolutions of $5 \mathrm{~nm}$ and span the visible spectrum from 410 to $720 \mathrm{~nm}$. The H\&E stained multispectral images were used as inputs to the proposed multispectral enhancement process while the Masson's trichrome stained images were used as reference for the visual evaluation of the enhanced images.

\subsection{Spectral transmittance data}

From the captured images we identified six different tissue components namely: nucleus, cytoplasm, red blood cells (RBC), collagen fiber, smooth muscle and duct. We obtained the H\&E stained spectral samples of these tissue components and the spectral samples of the white areas (areas which do not contain any tissue structures) to calculate for the principal component (PC) vectors $\mathbf{v}$, Eq. 4, and to investigate the spectral-error enhancement bands, $\boldsymbol{b}_{o}$, Eq. 10. Figure 2 shows the spectral samples projected onto the first three dominant principal component (PC) axes. The cluster plots provide an overview on the differentiation of these tissue components based on their H\&E staining color. Clearly, discrimination between tissue components which are stained with the same type of dye, such as the cytoplasm, smooth muscle and collagen fiber which are mainly stained with the eosin dye, is not very direct. We can observe from Fig. 2 that there are significant overlaps in the spectral clusters of these tissue components.

\subsection{Generation of the $N x N$ transformation matrix}

Linear mapping procedure is most effective when the spectral transmittance classes are linearly separable. Hence, to solve for the $N x N$ matrix $\mathbf{Q}_{g}$ in Eq. 6 we considered the spectral samples of nuclei, cytoplasm and $\mathrm{RBC}$ whose spectral clusters display clear-cut separations in the linear PCA space. In order for the white areas in the image, the areas which do not contain any tissue structures, to be preserved in the enhancement result, we also considered the spectral samples of the white areas in the mapping. The $N x C, N=63$, $C=4$, matrix, $\mathbf{F}$, in Eq. 6 contains the average $\mathrm{H} \& \mathrm{E}$ stained spectral samples of the nuclei, cytoplasm, and $\mathrm{RBC}$, and that of the white area, and matrix $\mathbf{G}$ contains the spectra of their target colors. The color patches in Fig. 4 demonstrate the original and target colors of these tissue components. The color patches at the leftmost panel demonstrate their original colors, while the two groups of color patches displayed at the right panel illustrate the tissue components' target colors. The first group of target colors was specified based on the Masson's trichrome staining colors of the tissue components. The second group of target colors, on the other hand, was freely specified by the user. The corresponding transmittance spectra of the first group of target colors were obtained from the Masson's trichrome multispectral images that were captured, while the transmittance spectra for the second group were calculated from the specified color values [7]. The transformation matrices using these sets of target colors were calculated using Eq. 6 wherein the pseudoinverse $\boldsymbol{F}^{+}$is determined using the function in Matlab [22]. In the following discussions, we refer to the transformation matrices derived using the first and second group of target colors as $\mathbf{Q}_{g a}$ and $\mathbf{Q}_{g b}$, respectively.

\subsection{Determination of the spectral residual-error}

The spectral data set that we used to derive the principal component (PC) vectors v, Eq. 4, to determine the spectral residual-error of a multispectral pixel consisted of the spectral samples of the non-collagen fiber 


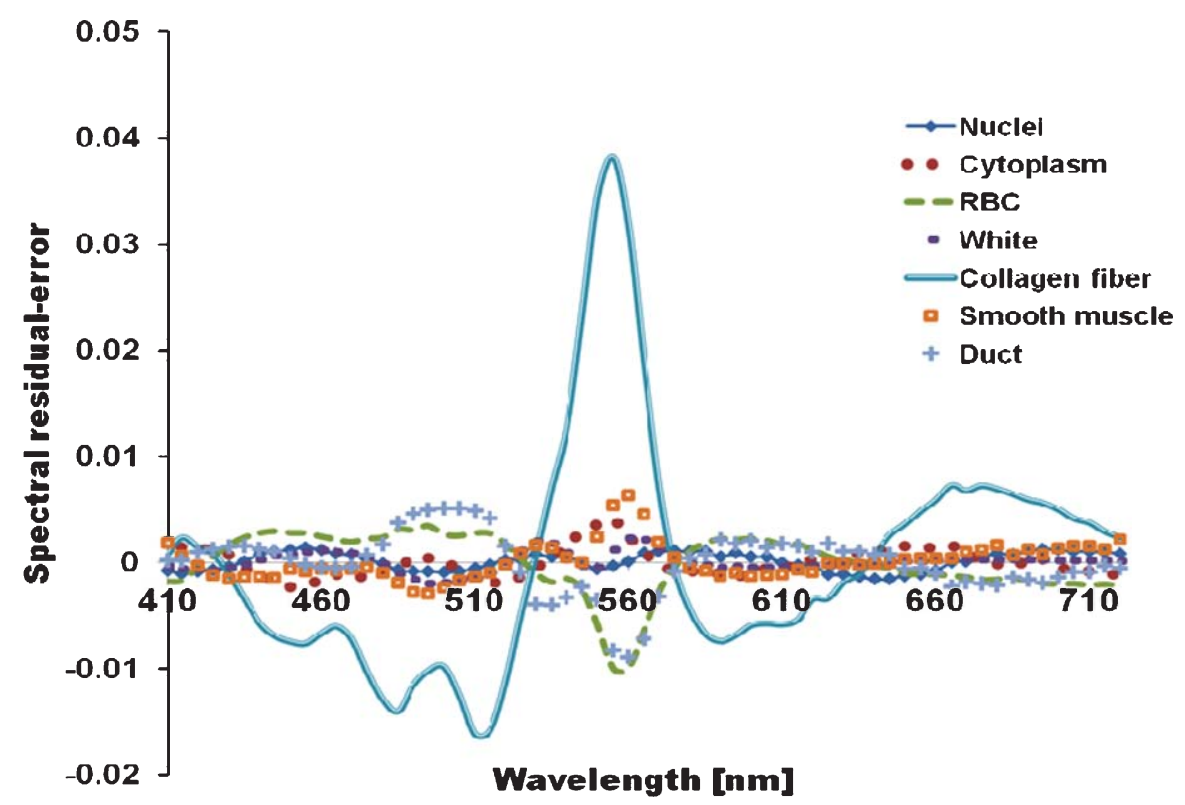

(a)

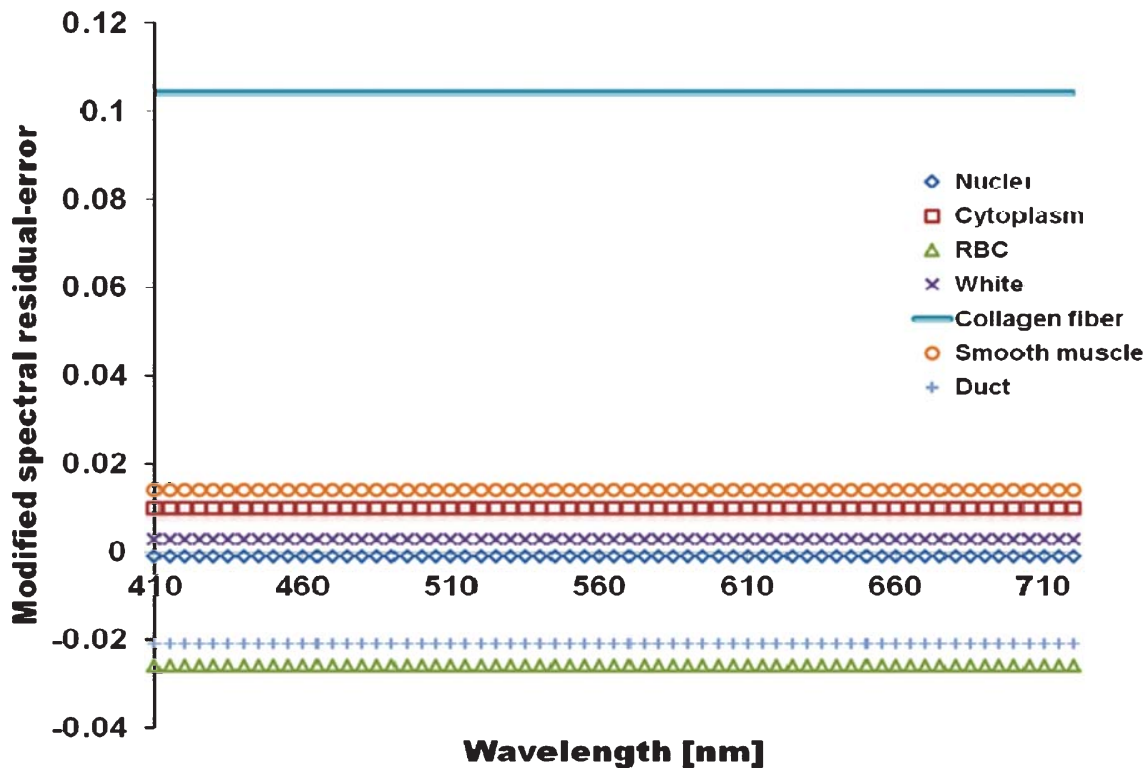

(b)

Fig. 4. The plots illustrate the average spectral residual-error of the different tissue components. (a) Original form of the spectral residual-error. Here, we can observe variations in the error values at different bands; (b) Modified form of the spectral residual-error. Here, the error values were mapped to a constant, which is the sum of the spectral residual-error at bands $550-560 \mathrm{~nm}$. These bands correspond to the bands at which the error of the collagen fiber is at its maximum.

tissue structures, which include the nuclei, cytoplasm, RBC, smooth muscle, and duct. The spectral samples of the white areas were also included. This implies that the derived principal component vectors could well estimate the spectra of the non-collagen fiber tissue structures but not the spectrum of the collagen fiber itself. We used five principal component (PC) vectors, i.e., $\mathbf{v}_{j}, j \leq 5$, to re-construct the original spectrum 

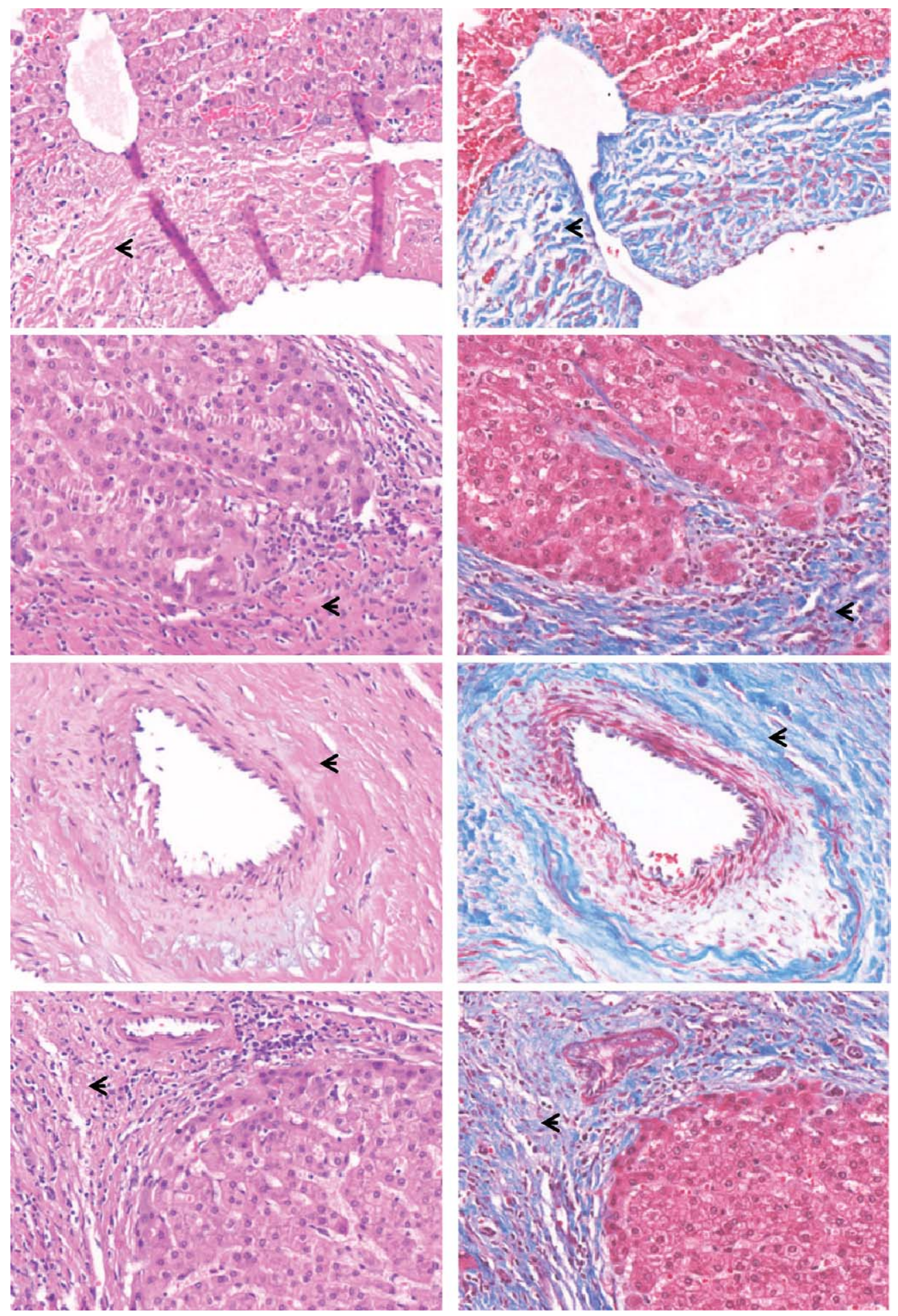

Fig. 5. RGB images of the hematoxylin and eosin (H\&E) stained images along with their Masson's trichrome stained counterparts. Images on the first column correspond to the H\&E stained images and images on the second column illustrate how the different tissue structures in the H\&E stained images appear in a Masson's trichrome stained tissue section. 
of the pixel using Eq. 3. This number was based on the experimental results reported in [8]. To determine the modified spectral-residual error using Eq. 8, we first determined the sets of bands for enhancement, $\mathbf{b}_{o}$, from the spectral error configuration of the collagen fiber. Figure $4 \mathrm{a}$ displays the average spectral residual-errors of the different tissue components in their original configurations. We selected the bands at which there is a significant difference between the spectral error's magnitude of the collagen fiber and the rest of the tissue components, and these correspond to: $\boldsymbol{b}_{o}=\{550,555,560 \mathrm{~nm}\}$. Figure $4 \mathrm{~b}$ demonstrates the effect of re-assigning the spectral error values to the result of Eq. 8. We can observe that the magnitude of the modified spectral residual-error, as shown in Fig. 4b, is constant across the different spectral bands, which is in contrast to the original spectral residualerror displayed in Fig. 4a where there are obvious variations in the error values between different spectral bands.

\subsection{Enhancement of multispectral images}

Figure 5 displays the original H\&E stained images. The Masson's trichrome stained images on their right serve as reference for the visualization of the collagen fibers, which are denoted by arrows, in the enhanced image. In the enhancement, the user-defined bands for enhancement $\boldsymbol{b}_{d}$ were set to 480 to $520 \mathrm{~nm}$ at a step of $5 \mathrm{~nm}$. The spectral residual-errors at these bands were weighted by a factor of $k=10$, and the weighting factors for the rest of the errors were set to $k=0$. The enhanced H\&E stained images are presented in Fig. 6. The enhanced images at each column represent the enhancement results for a particular transformation matrix $\mathbf{Q}_{g}$ : (i) $\mathbf{Q}_{g}=\mathbf{I}$, where $\mathbf{I}$ is an identity matrix; (ii) $\mathbf{Q}_{g}=\mathbf{Q}_{g a}$; and (iii) $\mathbf{Q}_{g}=\mathbf{Q}_{g b}$; the derivation of the $\mathbf{Q}_{g a}$ and $\mathbf{Q}_{g b}$ matrices are discussed in Section 3.3. The different sets of enhanced images show improved visualization of the collagen fiber when compared to their original H\&E stained images in Fig. 5. The results further illustrate the effect of the transformation matrices to the enhancement results. For instance, the color of the enhanced images on the second column share similarity to the Masson's trichrome stained images in Fig. 5, especially the color impression of the background, i.e., non-collagen fiber areas; and the enhanced images on the third column show better discrimination between the nuclei, cytoplasm, and RBC.
Table 1

The spectral bands at which the spectral residual-error were weighted with non-zero weighting factors

\begin{tabular}{lcc}
\hline $\begin{array}{l}\text { Enhanced } \\
\text { images }\end{array}$ & $\begin{array}{c}\text { Bands for enhancement, } \boldsymbol{b}_{d} \\
(\mathrm{~nm})\end{array}$ & $\begin{array}{c}\text { Weighting } \\
\text { factor, } k\end{array}$ \\
\hline Fig. 7a & $480-500$ & 30 \\
Fig. 7b & $480-500 ; 520-530$ & $20 ; 10$ \\
Fig. 7c & $520-530$ & 20 \\
Fig. 7d & $550-560$ & 20 \\
\hline
\end{tabular}

The enhancement color of the collagen fiber can be varied by varying the combination of the spectral bands in $\boldsymbol{b}_{d}$ or by varying the value of weighting factor assigned to each band in $\mathbf{W}$. This is being demonstrated by the enhanced images in Fig. 7. The transformation matrices $\mathbf{Q}_{g a}$ and $\mathbf{Q}_{g b}$ were used to generate the enhanced images on the first and second rows, respectively. The bands for enhancement for each enhanced image are listed in Table 1. Here, we can see that the user can freely change the color of the object of interest, which is the collagen fiber. The enhancement color the collagen fiber is associated to the spectral color of the bands specified in $\boldsymbol{b}_{d}$.

\subsection{Color discrimination evaluation}

We used the CIELAB color difference as metric to evaluate the color discrimination between the different tissue components [7]. We randomly extracted spectral samples for the different tissue components from the 13 multispectral H\&E images that we captured and applied spectral enhancement using Eq. 13. Table 2 shows the average Euclidian distance between the centers of the $L a b$ color vectors of the different tissue components for the three different cases of transformation matrices. It is noted that the original color difference between the collagen fiber and smooth muscle is less than 5. This implies that from an H\&E stained tissue section these tissue structures are perceptually similar. It is shown that application of spectral enhancement helps improves the color discrimination between these two structures. Furthermore, the color difference values presented in Table 1 illustrate the effectiveness of introducing the transformation matrix $\mathbf{Q}_{g}$ to the enhancement formulation in the overall multispectral enhancement result. By appropriately designing the transformation matrix we can further improve the visualization and color discrimination of both the collagen fiber and its background structures. 

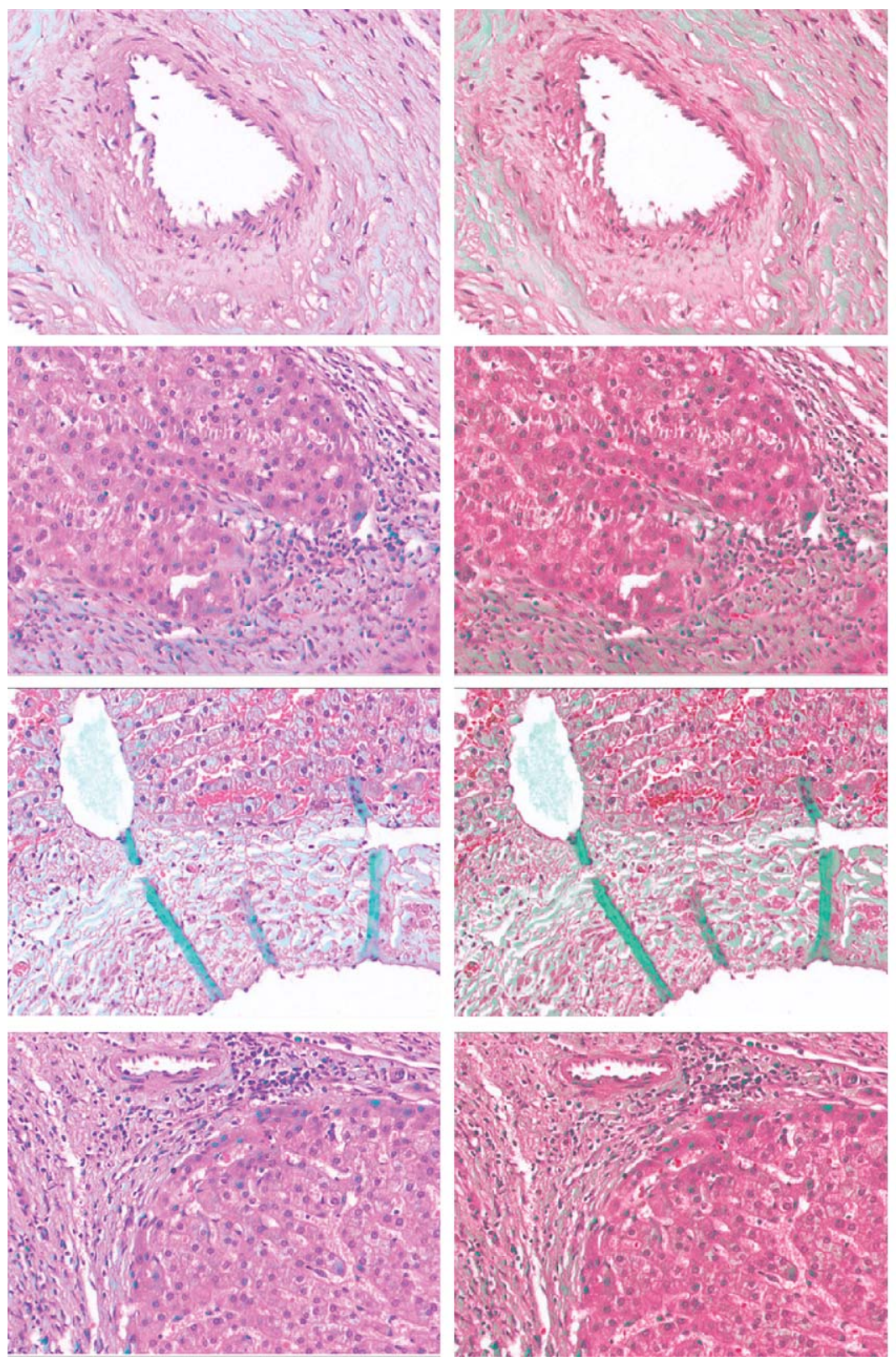
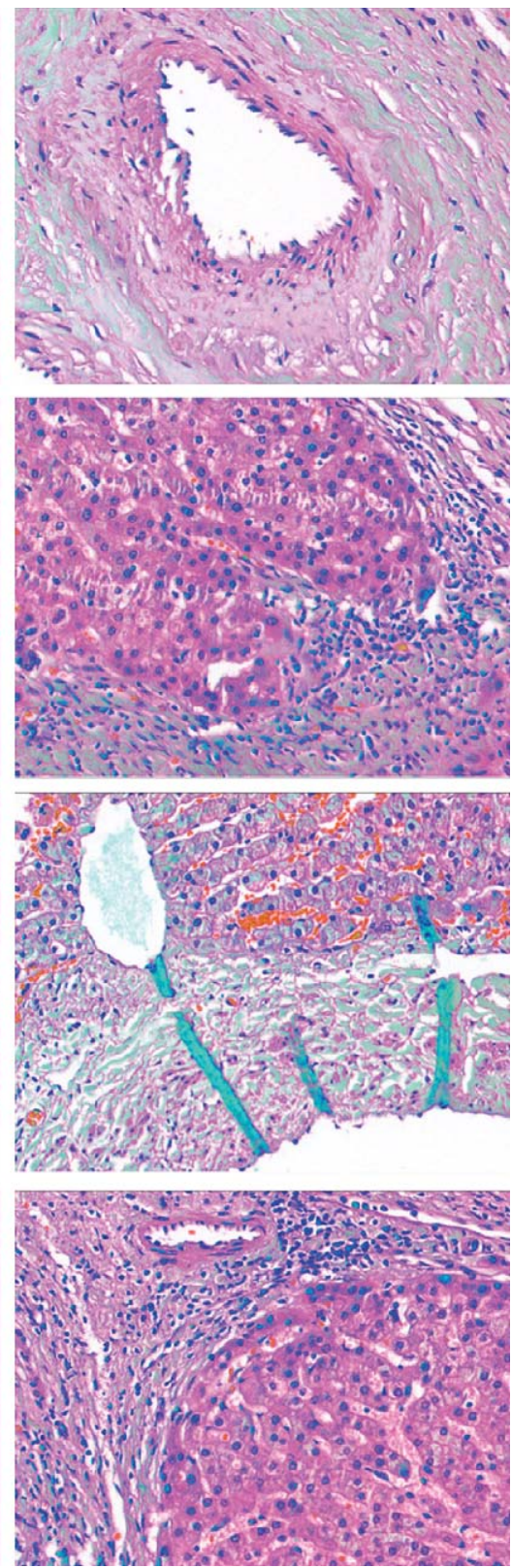

Fig. 6. Enhancement results illustrating the effect of using different designs of transformation matrices. The bands for enhancement were set to $520-530 \mathrm{~nm}$ and the weighting factors were set to $k=20$ at these bands. Enhanced images at the first column demonstrate the enhancement results when $\mathbf{Q}_{g}$ is set to an identity matrix; enhanced images at the second and third columns illustrate the effect of the transformation matrices derived using the target colors illustrated in Fig. 4b, c.

\section{Discussion}

In this work we have introduced a concept of multispectral enhancement which allows the user to vary the enhancement color of the object of interest as well as correct or modify the color of the background struc- tures to the colors envisioned by the user. The option to modify the color of the background tissue structures is beneficial especially when the tissue sections from which the images were captured are poorly stained. Results of our experiments show that by appropriately designing the transformation matrix, the matrix 


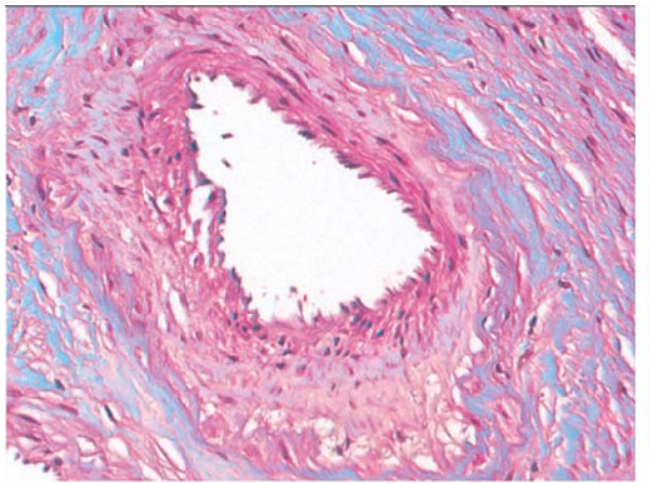

(a)

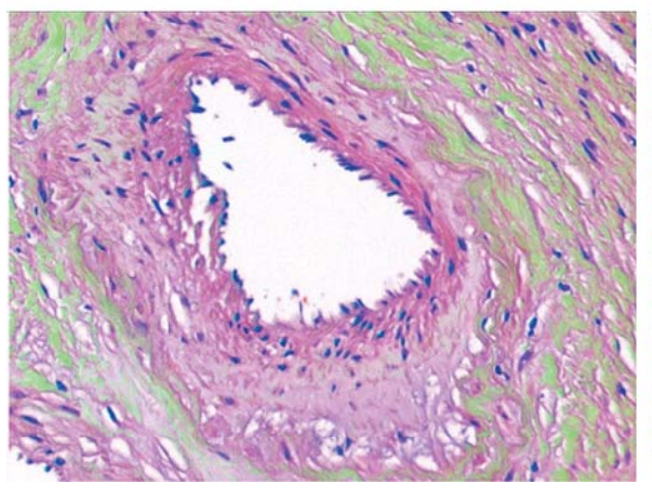

(c)

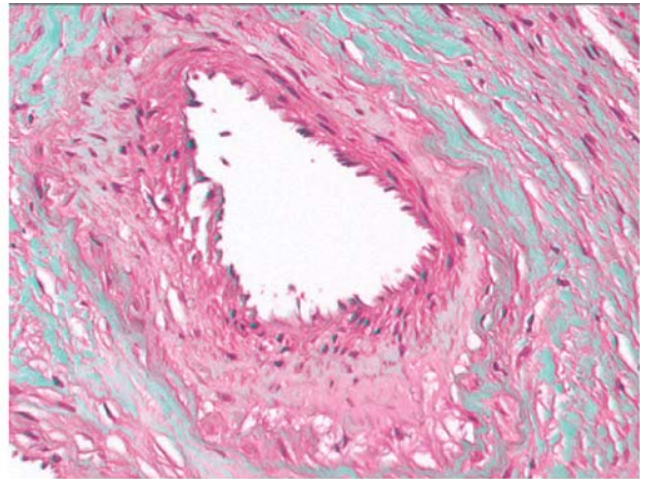

(b)

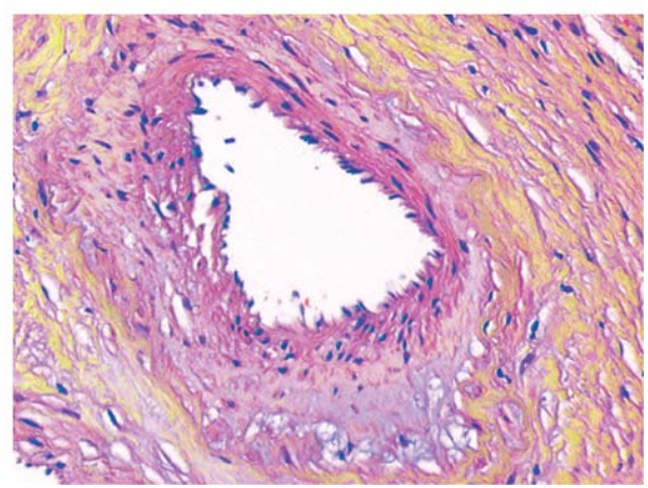

(d)

Fig. 7. Enhancement results illustrating the effect of choosing different bands for enhancement. The transformation matrix used to obtain these images was $\mathbf{Q}_{g b}$, i.e., using target colors illustrated in Fig. 4b. The bands for enhancement used to produce these images are reported in Table 1 . By choosing different bands for enhancement the enhancement color of the object of interest, which is at present the collagen fiber, can be varied.

Table 2

Average color difference, $\Delta E_{a b}$, between the different tissue components for the different design of transformation matrices

\begin{tabular}{|c|c|c|c|c|}
\hline \multirow[t]{2}{*}{ Tissue components being compared } & \multirow[t]{2}{*}{ Original } & \multicolumn{3}{|c|}{ Transformation matrices used $\left(\boldsymbol{b}_{d}=[520-530 \mathrm{~nm}], k=20\right)$} \\
\hline & & $\mathbf{Q}_{g}=\mathbf{I}$ & $\mathbf{Q}_{g}=\mathbf{Q}_{g a}$ & $\mathbf{Q}_{g}=\mathbf{Q}_{g b}$ \\
\hline Nuclei \& Cytoplasm & 34.64 & 35.35 & 33.28 & 57.02 \\
\hline Nuclei \& RBC & 47.92 & 61.13 & 52.56 & 125.19 \\
\hline Cytoplasm \& RBC & 27.10 & 34.73 & 43.039 & 84.97 \\
\hline Fiber \& Cytoplasm & 7.52 & 36.15 & 39.62 & 55.84 \\
\hline Fiber \& Smooth muscle & 4.77 & 51.67 & 62.01 & 66.86 \\
\hline
\end{tabular}

*Number of spectral samples: Nuclei -30700; Cytoplasm -20400; RBC -7500; Fiber -31200; muscle -7200.

used to convert the original spectrum of the multispectral pixels to the target spectral configuration, better visual perception of the different tissue structures can be achieved. The multispectral enhancement method proposed in [7] which was also extended in [23] to enhance the collagen fiber areas also allows the user to define the color for enhancement. However, the method is most effective only when the hues of the background pixels are similar, which is not true for the multispectral enhancement method proposed in this paper.

Figure 8a shows magnified areas cropped from the original H\&E and Masson's trichrome images. The 

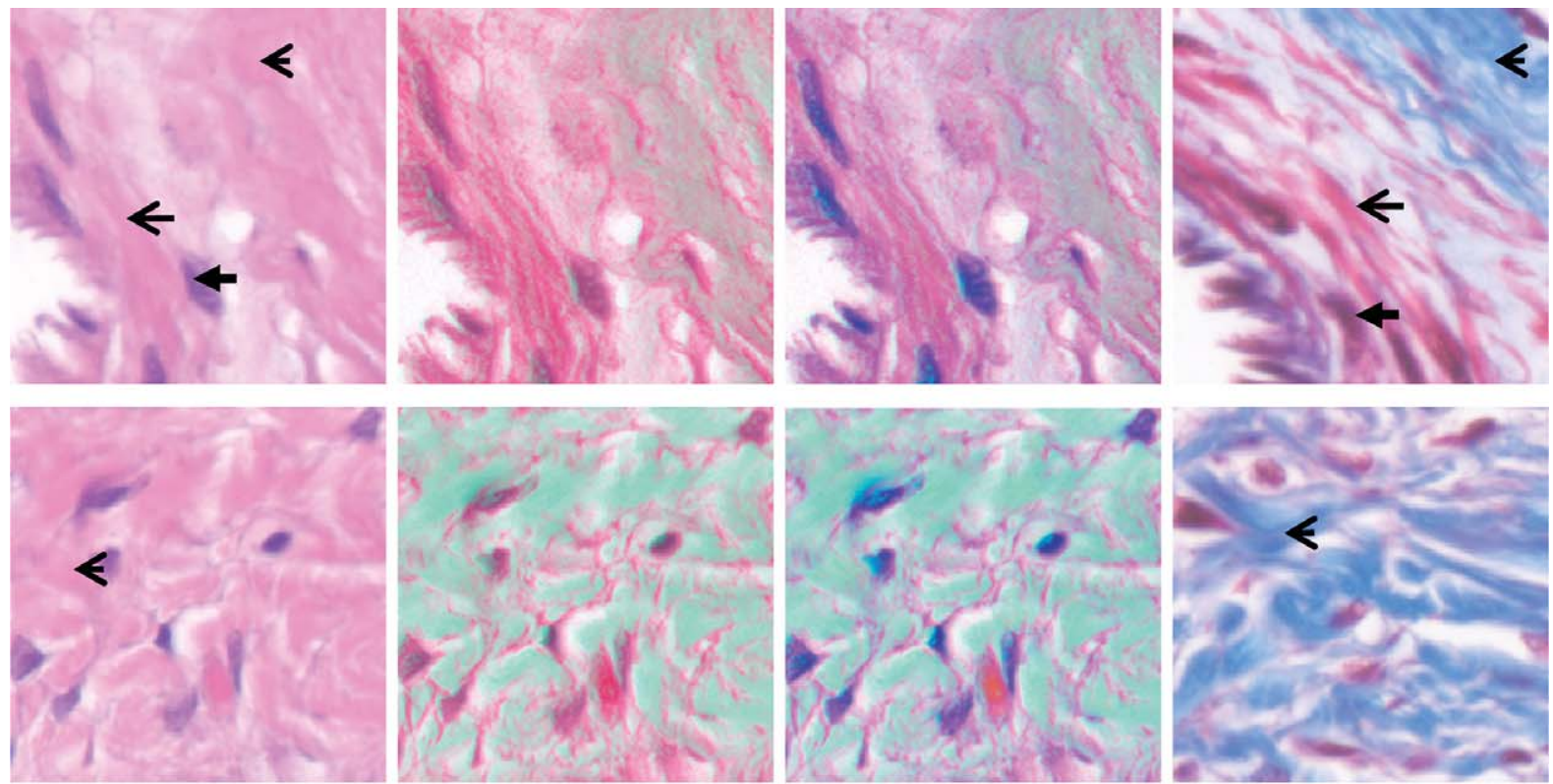

(a)
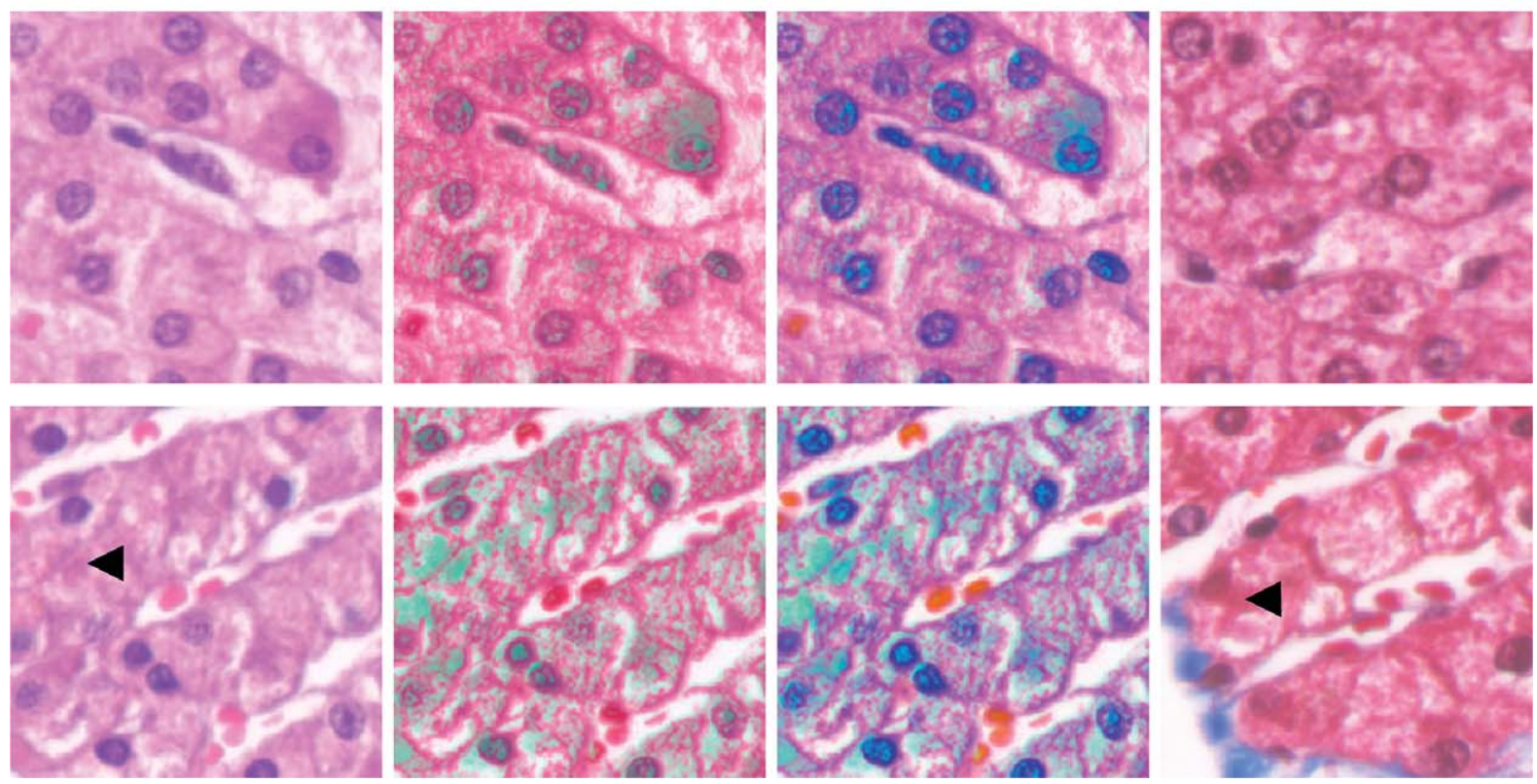

$\leftarrow$ Collagen fiber

(b)

$\leftarrow$ Nucleus

\section{Cytoplasm}

\section{$\leftarrow$ Smooth muscle}

Fig. 8. Areas cropped from the original H\&E stained and enhanced images. (a) These images show the effectiveness of the present enhancement scheme to differentiate collagen fibers and smooth muscles which are originally not clearly discriminated from an H\&E stained tissue section. This set of images also show the variation of the response of the collagen fiber areas to the enhancement, i.e., some areas are more saturated than others. (b) These images show areas which are mislabeled in the present enhancement. Cytoplasm pixels were mislabeled to Collagen fiber. 
leftmost and rightmost images respectively illustrate the staining patterns of H\&E and Masson's trichrome stains. We can observe that while the smooth muscle and collagen fiber areas display indistinct $\mathrm{H} \& \mathrm{E}$ staining patterns, they are prominently discriminated in the Masson's trichrome stained image. The digitally enhanced H\&E stained images at the center panels show similar color discrimination as the Masson's trichrome stained image wherein the collagen fiber and smooth muscle areas also display distinct colors. It has been shown in [8] that the spectral difference between the smooth muscle and collagen fiber lies in their differing reaction to eosin stain. The spectral bands at which the spectral residual-error of the collagen fiber has large values are correlated to the eosin stain absorption bands, Fig. 4a. One of the issues pointed by authors in [8] in the simulation of the Masson's trichrome staining is the color of the nuclei which appear reddish in the digitally stained images. The present experimental results show that designing the transformation matrix $\mathbf{Q}_{g}$ using the spectral samples that are linearly separable improves the enhanced color of the nuclei.

An important aspect in any enhancement algorithms is consistency in delivering the desired results regardless of the image condition. For the same weighting factor value $k$ we can observe differing responses of the collagen fiber areas to the enhancement as noted by the variation in their color saturation, Fig. 8a. We can also observe that some pixels belonging to the cytoplasm were mis-detected to be part of the collagen fiber in that they display the enhancement color assigned to collagen fiber, Fig. $8 \mathrm{~b}$. This could be the underlying reason why the color difference between the cytoplasm and collagen fiber is smaller in comparison to the color difference between the collagen fiber and smooth muscle after enhancement, Table 2. This result also shows that there is a greater variance in the spectra of the pixels belonging to cytoplasm areas. It is thought that the current results can be improved further by: (i) incorporating information on the staining condition of the multispectral pixel in the definition of the weighting matrix; (ii) integrating statistical properties of the multispectral pixels to the enhancement formulation; and (iii) increasing the number of spectral samples to encompass the staining variations of the different tissue sections. Moreover to address variations in H\&E staining protocols, staining correction method such as the one proposed in [24] could be employed to pre-process the images.

\section{Conclusion}

We have presented a multispectral enhancement methodology whereby we could effectively visualize the color differences between tissue structures which display similar H\&E staining patterns. Moreover, the options in the current multispectral enhancement approach allow us to digitally simulate the effect of chemical staining, i.e., Masson's trichrome staining, by designing appropriately the transformation and weighing matrices. The viability of the method to provide clearer discrimination between the different tissue structures has been illustrated by the enhancement results of the H\&E stained images. The present enhancement methodology can be very useful to visualize tissue structures which are not emphasized by the original stain thereby reducing the cost of staining and the time to deliver a diagnosis. The technology of whole slide scanners is evolving. Integrating multispectral acquisition capability in the scanner designs are being looked into. In this case, the proposed multispectral enhancement can be incorporated into the scanners' image acquisition or image analysis software to deliver digitally enhanced H\&E images which possess improved visualization of the tissue structure of interest.

\section{Acknowledgment}

This work is supported in part by Olympus, Inc. Japan.

\section{References}

[1] D. Bratasanu, I. Nedelcu and M. Datcu, Interactive spectral band discovery for exploratory visual analysis of satellite images, IEEE J Selected Top Appl Earth Observations and Remote Sensing 5(1) (2012), 207-204.

[2] F. Joel, W.-M. Leong, S.-Y.A. Leong, et al., Basal Lamina Visualization Using Color Image Processing and Pattern Recognition, Appl Immunohistochem Mol Morphol 5(3) (2005), 273-277.

[3] D.S. Gareau, Feasibility of digitally stained multimodal confocal mosaics to simulate histopathology, J Biomed Opt 14(3) (2009), 034050.

[4] J. Bini, J. Spin, K. Nehal, V. Hazelwood, C. DiMarzio and M. Rajadhyaksha, Confocal Mosaicing microscopy of human skin ex vivo: Spectral analysis for digital staining to simulate histology-like appearance, J Biomed Opt 16(7) (2011), 076008. 
[5] P.A. Bautista, T. Abe, M. Yamaguchi, Y. Yagi and N. Ohyama, Digital staining of unstained pathological tissue samples through spectral transmittance classification, Opt Rev, 12(1) (2005), 7-14

[6] P.A. Bautista, T. Abe, M. Yamaguchi and N. Ohyama, Digital staining for multispectral images of pathological tissue specimens based on combined classification of spectral transmittance, Comput Med Imaging and Graph 29 (2005), 649-657.

[7] N. Hashimoto, Y. Murakami, P.A. Bautista, M. Yamaguchi, T. Obi, N. Ohyama, K. Uno and Y. Kosugi, Multispectral image enhancement for effective visualization, Opt Express 19(10) (2011), 9315-9329.

[8] P.A. Bautista and Y. Yagi, Digital simulation of staining in histopathology images: Enhancement and linear transformation of spectral transmittance, J Biomed Opt 17(5) 2012, 056013.

[9] C.M. Gilbert and A. Parawani, The use of multispectral imaging to distinguish reactive urothelium from neoplastic urothelium, J Pathol Inform 1 (2010), 23.

[10] I. Mansoor, C. Zalles, F. Zahid, K. Gossage, R.M. Levenson, and D.L. Rimm, " Fine-Needle Aspriation of Follicular Adenoma Versus Parathyroid Adenoma: The Utility of Multispectral Imaging in Differentiating Lesions With Subtle Cytomorphologic Differences,", Cancer 114(1) (2008), 22-26.

[11] R.M. Levenson, J. Beechem and G. Mcnamara, Spectral imaging in preclinical research and clinical pathology, Anal Cell Pathol 36 (2012), 1-23.

[12] K.E. McGrath, T.P. Bushnell and J. Palis, Multispectral imaging of hematopoietic cells: Where flow meets morphology, $J$ Immunol Methods 336(2) (2008), 91-97.

[13] K. Fujii, M. Yamaguchi, N. Ohyama and K. Mukai, Development of support systems for pathology using spectral transmittance-The quantification method of stain conditions, Conf Proc SPIE 4684 (2002), 1516-1523.
[14] L.L. Nuffer, P.A. Medvick, H.P. Foote, and J. Solinsky, Hyperspecptral/multispectral image enhancement for biological cell analysis, Cytometry 69A (2006), 897-903.

[15] M. Mitsui, Y. Murakami, T. Obi, M. Yamaguchi and N. Ohyama, Color enhancement in multispectral image using the karhunen-loeve transform, Opt Rev 12(2) (2005), 6975.

[16] N. Ohyama, K. Suzuki, T. Honda, J. Tsujiuchi, L. Ono, and S. Ikeda Digital, Processing of endoscopic color images, $O p t$ Commun 55(4) (1985), 242-247.

[17] S. Waheed, R.A. Motiff, Q. Chaudry, A.N. Young and M.D. Wang, Computer aided histoptahological classification of cancer subtypes, Proc. 7th IEEE Int Conf Bioinform Bioeng (2007), 503-508.

[18] T. Walter, P. Massin, A. Erginay, R. Ordonez, C. Jeulin and J-C Klein, Automatic detection of microaneurysms in color fundus images, Med Image Anal 11 (2007), 555-566.

[19] C.I. Sanchez, M. Garcia, A. Mayo, M. Lopez and R. Hornero, Retinal image analysis based on mixture models to detect hard exudates, Medi Image Anal 13 (2009), 650-658.

[20] H.F. Jaafar, A.K. Nandi, and W. Al-Nuaimy, Decision support system for the detection and grading of hard exudates from color fundus photographs, J Biomed Opt 16(11) (2011), 116001.

[21] K. Shinoda, Y. Murakami, M. Yamaguchi and N. Ohyama, Lossless and lossy coding for multispectral image based on sRGB standard and residual components, J Electron Imaging 20(2) (2011), 023003.

[22] Matlab Technical Computing Version R2800a.

[23] P.A. Bautista and Y. Yagi, Multispectral enhancement towards digital staining, Anal Cell Pathol 35(1) (2012), 51-55.

[24] T. Abe, Y. Murakami, M. Yamaguchi, N. Ohyama and Y. Yagi, "Color correction of pathological images based on dye amount quantification", Opt Rev 12(4) (2005), 293-300. 


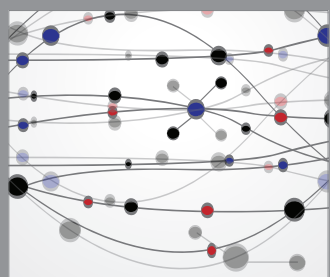

The Scientific World Journal
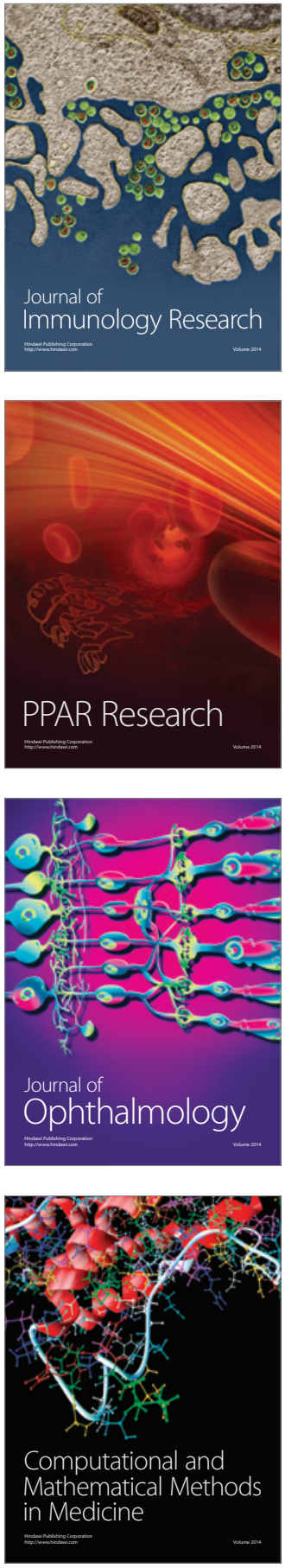

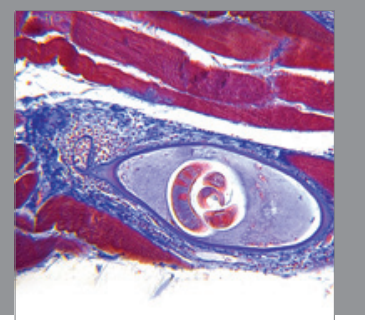

Gastroenterology

Research and Practice
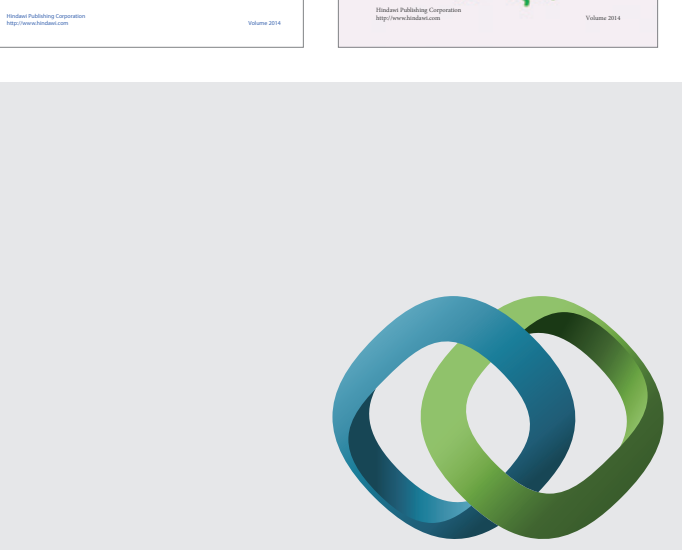

\section{Hindawi}

Submit your manuscripts at

http://www.hindawi.com
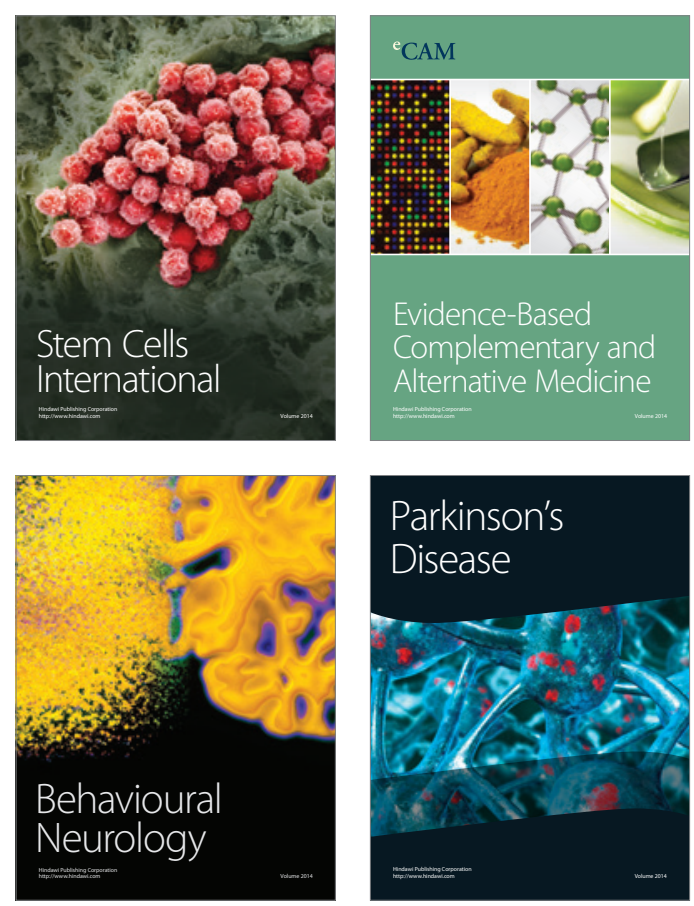

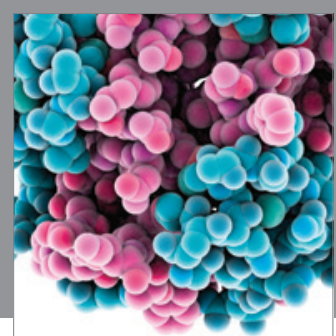

Journal of
Diabetes Research

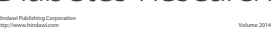

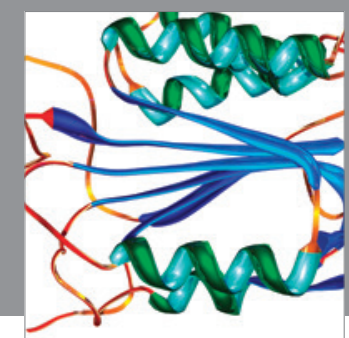

Disease Markers
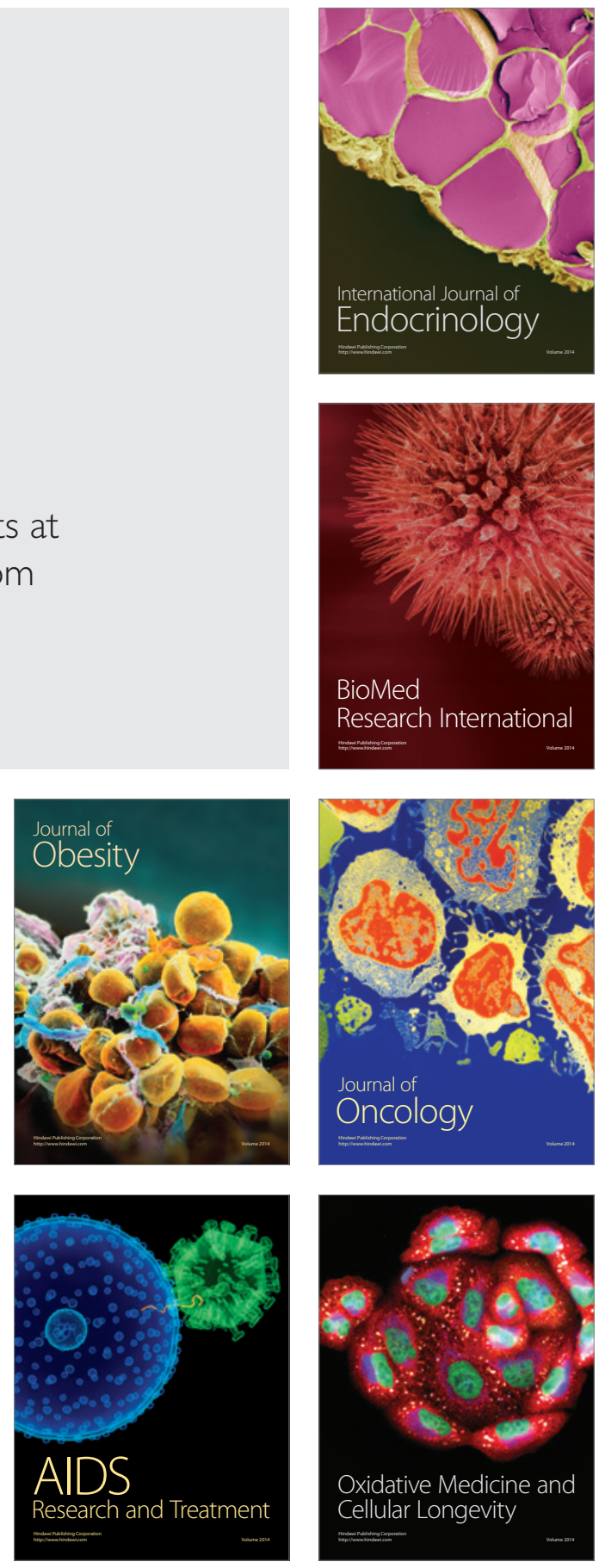